\title{
A sandpile experiment and its implications for self-organized criticality and characteristic earthquake
}

\author{
Naoto Yoshioka \\ Graduate School of Integrated Science, Yokohama City University, Seto 22-2, Kanazawaku, Yokohama 236-0027, Japan
}

(Received February 14, 2003; Revised July 7, 2003; Accepted July 8, 2003)

\begin{abstract}
We have performed an experiment in which a conical sandpile was built by slowly dropping sand onto a circular disk through a funnel with a small outlet. Avalanches (sand dropping off the disk) occurred, the size and the number of which were observed. It was seen that the behavior of avalanches (frequency-size distribution) was determined solely by the ratio of grain size to disk size, which is consistent with earlier studies. We categorize the behavior into three types: (1) the self-organized criticality (SOC) type (obeying Gutenberg-Richter's law), (2) the characteristic earthquake (CE) type where only large avalanches are almost periodically generated, and (3) the transition type. The transition from SOC to CE type drastically occurs when the ratio of grain diameter to disk radius is reduced to about 0.02 . The underlying mechanism to cause the transition is considered. Results of simulation by cellular automaton models, an experimental result showing that a conical pile has a stress dip near its center, and a two-dimensional simulation building up a conical pile, all suggest that the transition occurs due to a change in stress profile inside and near the surface of the pile. Although we are unfortunately not able to understand the detailed mechanism at the present stage, it seems very important to further investigate the underlying physics of the transition because it presumably provides us a clue to understand the mechanism of the periodicity of large characteristic earthquakes and may open a way for earthquake prediction.
\end{abstract}

Key words: Sandpile, self-organized criticality, characteristic earthquake.

\section{Introduction}

Since Bak, Tang and Wiesenfeld (1987, 1988, referred to as BTW model hereafter) proposed the concept of selforganized criticality (SOC), the Gutenberg-Richter's power law (G-R law) has been regarded as a typical natural example of SOC (Bak and Tang, 1989). The frequency-size distribution of earthquakes exhibits a straight line on a log-log plot (power law) and has no characteristic scale.

On the other hand, it has been pointed out that individual faults tend to generate essentially same size or characteristic earthquakes (CE) which have a relatively narrow range of magnitude (Schwartz and Coppersmith, 1984). In fact, the large earthquakes along the Nankai trough, Japan, seems to have periodically occurred with almost same magnitudes (Ando, 1975; Shimazaki and Nakata, 1980; Kumagai, 1996). The earthquake frequency-size distribution including these large earthquakes does not satisfy the G-R law (Wesnousky et al., 1983) and hence seems to conflict with the concept of SOC.

This problem is related to the question whether earthquake prediction is possible or inherently not. If the occurrence of all earthquakes obeys a power law, earthquakes may not be predicted because every fault is in a critical state and any small earthquake has some probability of growing into a large earthquake (Geller et al., 1997). However large characteristic earthquakes occur more frequently than would be expected by an extrapolation of linear recurrence relation-

Copy right(C) The Society of Geomagnetism and Earth, Planetary and Space Sciences (SGEPSS); The Seismological Society of Japan; The Volcanological Society of Japan; The Geodetic Society of Japan; The Japanese Society for Planetary Sciences. ship from lower magnitude earthquakes (Fig. 1). This indicates that the generation process of characteristic earthquakes is somewhat different from that of intermediate and small earthquakes. The periodicity of characteristic earthquakes may leave room for seeking a way of earthquake prediction.

A number of numerical studies have been performed after the appearance of BTW model. For example, Kadanoff et al. (1989) performed an extensive numerical study of the sandpile model and found that the frequency-size distribution of avalanches obeys a power law. By varying the sizes of the system, they investigated the scaling properties of the models. Barriere and Turcotte (1994) carried out a simulation by a cellular automaton model in which they considered a grid of cells with a fractal distribution of sizes. The frequencysize statistics was found to satisfy the G-R law. Carlson and Langer (1989a, b) presented a slider-spring model for fault dynamics (CL model), which was introduced originally by Burridge and Knopoff (1967). They found that the model generated events of all sizes which obeyed a powerlaw frequency-size distribution. Motivated by CL model, Nakanishi $(1990,1991)$ presented a cellular-automaton version of the slider-spring model and showed that the model exhibited quite similar behavior to CL model. Ito and Matsuzaki (1990) also presented a cellular automaton model in which the effect of plasticity was taken into account. They also modified the model so that every earthquake may trigger aftershocks. The results all shows a power-law frequencysize distribution. The model calculations using various cellular automaton models clearly show SOC properties. 


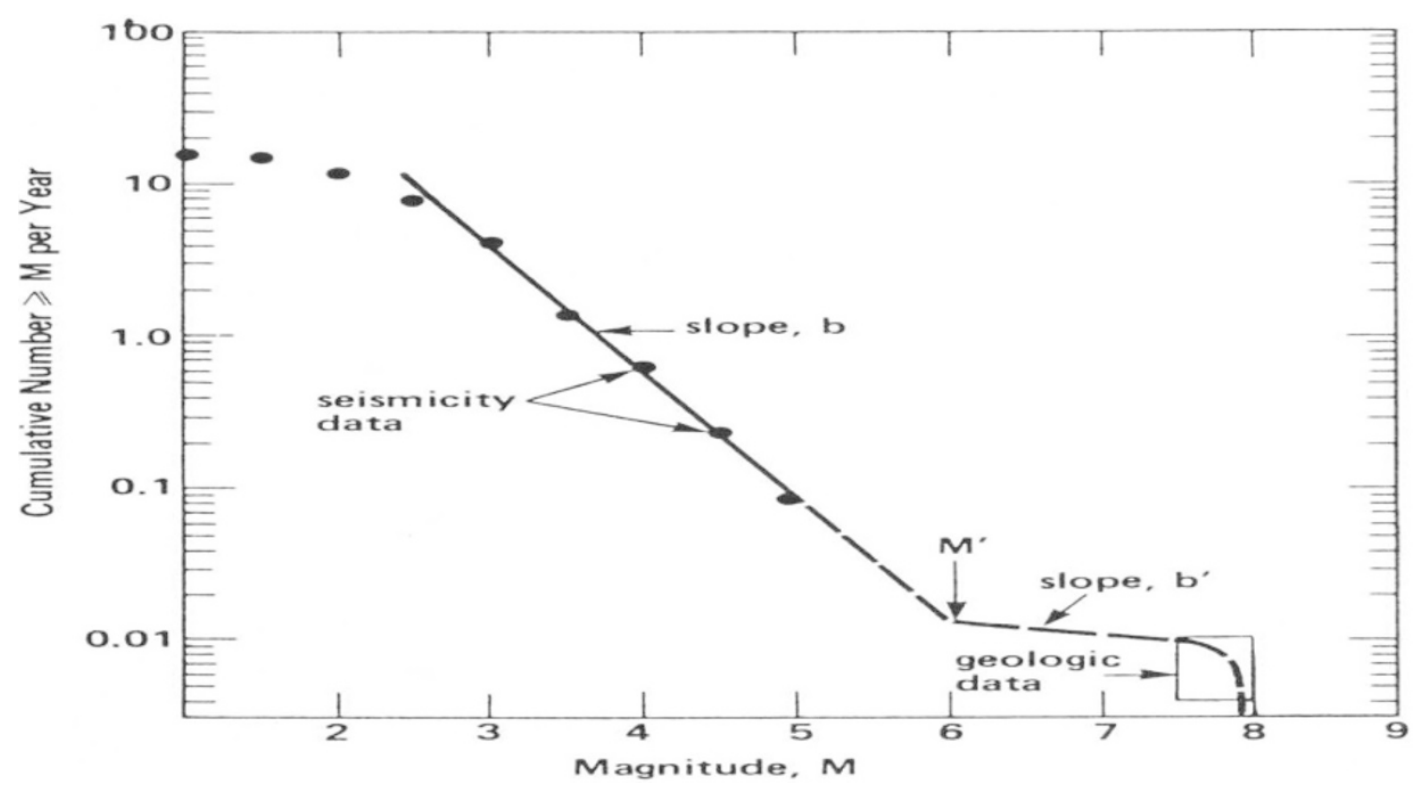

Fig. 1. Diagrammatic cumulative frequency-magnitude recurrence relation ship for an individual fault of fault segment (after Schwartz and Coppersmith, 1984).

Laboratory experiments using real sand have also been carried out. The results, however, did not show SOC-type behavior in contrast to the numerical experiments. Turcotte (1999) states in a review of SOC that experimental results have been quite ambiguous. For example, Jaeger et al. (1989) carried out an experiments in which a semicircular drum ( $5 \mathrm{~cm}$ radius) filled with sand (grain diameter $0.54 \mathrm{~mm}$ ) was slowly and continuously inclined. They measured the flow of sand over the edge of the drum. The results showed no power-law distribution in contradiction to BTW model and rather exhibits very regular spikes of large avalanches. They interpreted the results as a hysteresis effect whereby the pile's slope angle may exceed the angle of repose before a major avalanche. Evesque (1991) carried out an experiment using a rotating drum and obtained similar results of Jaeger et al. (1989).

Held et al. (1990) made a very sophisticated experiment in which a conical sandpile was built on a circular disk by adding sand grains one by one onto the apex of the sandpile. If an avalanche occurred in any scale, the addition of sand was stopped and the size of the avalanche was recorded. This process was automatically repeated. For sufficiently small sandpiles (less than 50 grain diameter), the size-frequency distribution was found to obey a power-law distribution and to show finite-size scaling. However large sandpiles (on the order of 100 grain diameter) no longer showed the finitesize scaling but rather generated periodic large avalanches. Similar results were obtained by Rosendahl et al. (1993) who carried out a sandpile experiment with the apparatus and techniques similar to those of Held et al. (1990). Rosendahl et al. (1994) discussed the possibility of prediction of a major large avalanche based on the change in the rate of small avalanche activity prior to it.

It seems that experiments by a rotating drum technique tend to produce a quasi-periodic large avalanches. The use of glass beads with small diameter compared with the drum radius may accelerate this tendency. The experiment building up a sandpile on a disk by adding sand grains is much more suitable for investigating SOC properties (Held et al., 1990).

We also performed a sandpile experiment using real sand grains to confirm the results of others mentioned above. Our experimental method is basically similar to that of Held et al. (1990) and Rosendahl et al. (1993). In this paper, we present the result of the experiment and consider its implication for SOC and characteristic earthquake.

\section{Experiment \\ 2.1 Experimental method}

Figure 2(a) shows the experimental setup. Beach sands (washed with water and dried at room temperature) were dropped from the hopper, bifurcated 7 times, slowed down by two funnels and slowly added on a circular disk (Fig. 2(b)). The flow rate of sand was $10-20 \mathrm{mg} / \mathrm{s}$ which was affected by slight inclination of the bifurcation jigs. In one experimental run, the fluctuation in flow rate was less than $10 \%$.

The weight of the sandpile was measured by a digital balance with the accuracy of $1 \mathrm{mg}$, and sent to a computer with the sampling frequency of $2.5 \mathrm{~Hz}$. Sandpiles with base diameters from 2 to $8 \mathrm{~cm}$ were studied. We used two kinds of sands with diameters ranging from 0.5 to $1 \mathrm{~mm}$ (sand 1) and ranging from 0.25 to $0.5 \mathrm{~mm}$ (sand 2). The average weight of the sand grains is $0.84 \mathrm{mg}$ for sand 1 and $0.14 \mathrm{mg}$ for sand 2.

The sand grains were dropped on the apex of the sandpile. However it was observed that they did not necessarily rest on the top of the sandpile, but rolled down slightly and settled somewhere on the slope. We also observed that during the addition of sand grains, some grains at rest on the slope began to roll down the slope and stop without dropping off the disk. We did not regard the case as an avalanche because 


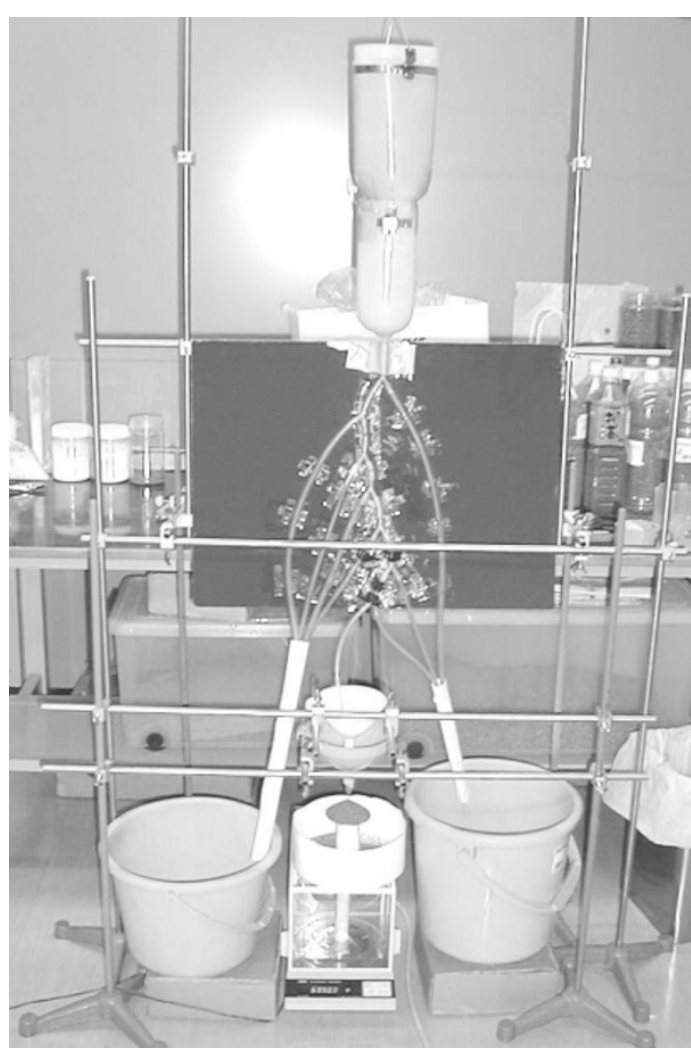

(a)

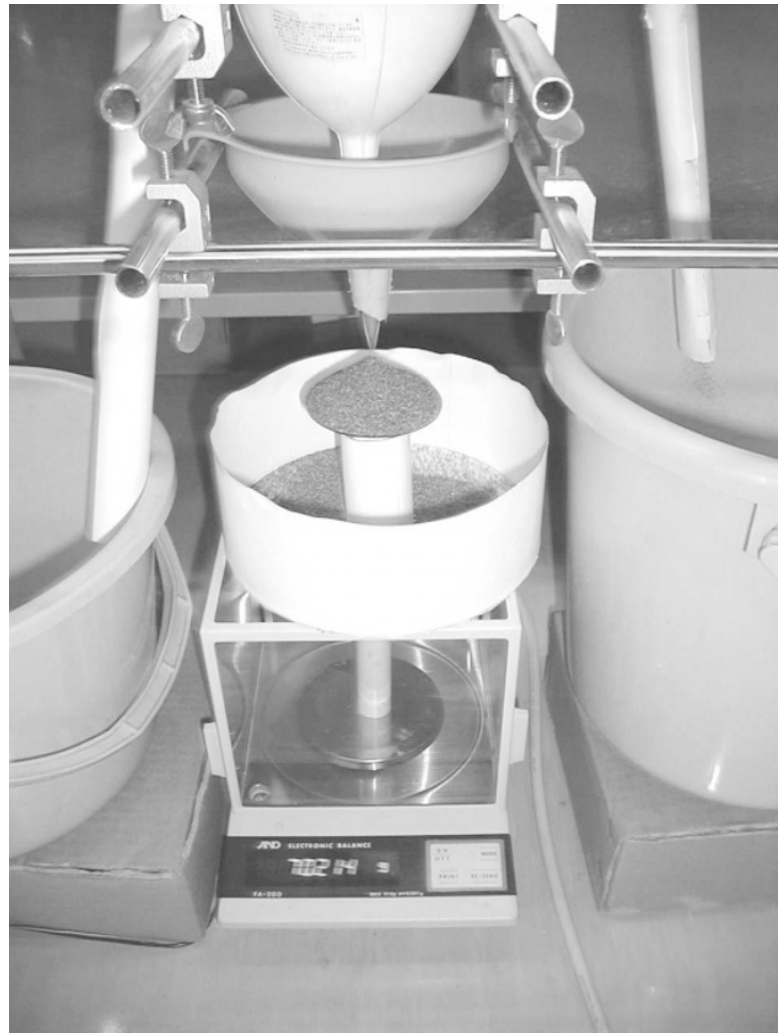

(b)

Fig. 2. Experimental setup. Beach sands were slowly dropped on a circular disk, and the total weight of the sandpile was continuously measured (see text).

the weight of the sandpile did not affected by this small landslide.

The experimental conditions which we suspected to affect the experimental results were humidity $(30-65 \%)$, the distance between the outlet of the lower funnel and the top of the sandpile $(1-4 \mathrm{~cm})$, and the diameter of funnels outlet (2-4 mm). However they gave no remarkable effect on the results.

\subsection{Counting avalanches}

Because the input and the output of sand grains onto/from the disk occurs simultaneously, we need to analyze the observed weight curves of sandpile to count the number and the size of avalanches. Figure 3 shows the counting procedure. Figure 3(b) shows the enlarged portion of the inlet in Fig. 3(a). The inclined dotted lines show the flow rate of this experiment (that is, if no grain drops off the disk, the curve of weight should follow the line). Since we observed that a small amount of sand grains continuously dropped off the disk after the steady state was reached (especially in small disks), we do not recognize such a steady flow as an avalanche. Here we expediently define an avalanche as a reduction in the total weight. For example, in the process from $a$ through $c$ in Fig. 3(b), the weight of the sandpile increases, but the increasing rate is less than the flow rate line. This is not recognized as an avalanche. The same situation is seen from $e$ to $f$ and from $h$ to $i$. We may underestimate the number of small avalanches due to this procedure. From $c$ through $e$, the weight is continuously decreasing. We recog-

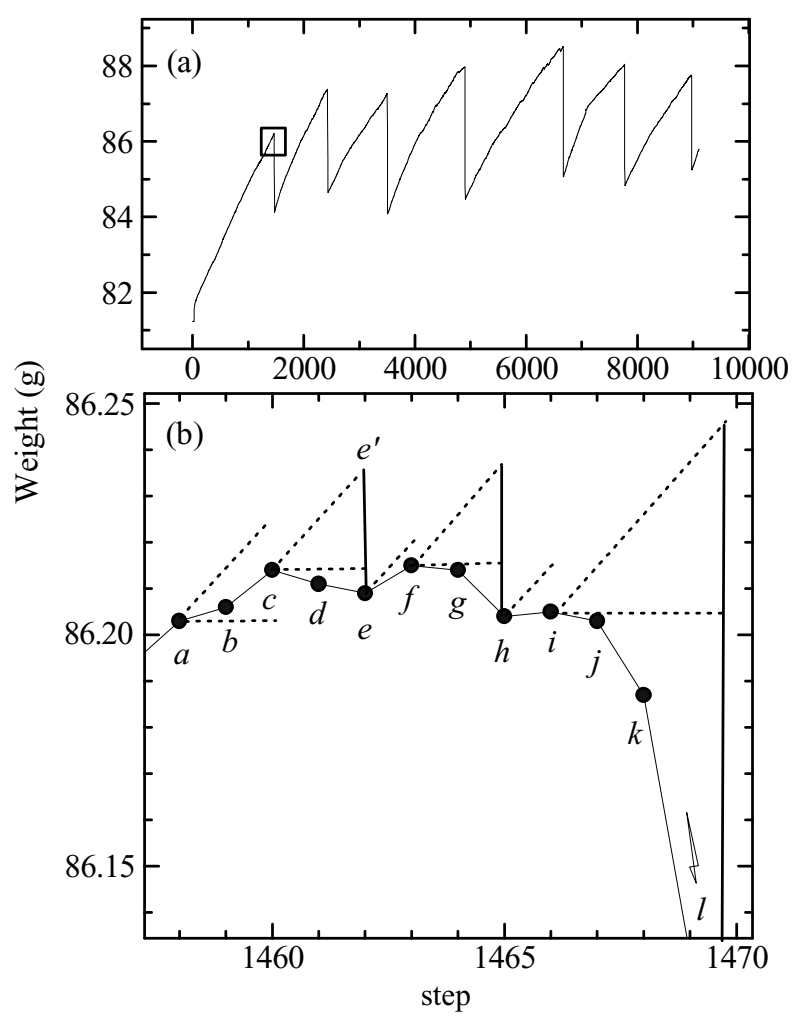

Fig. 3. Counting procedure of the number and the size of avalanches (see text). 


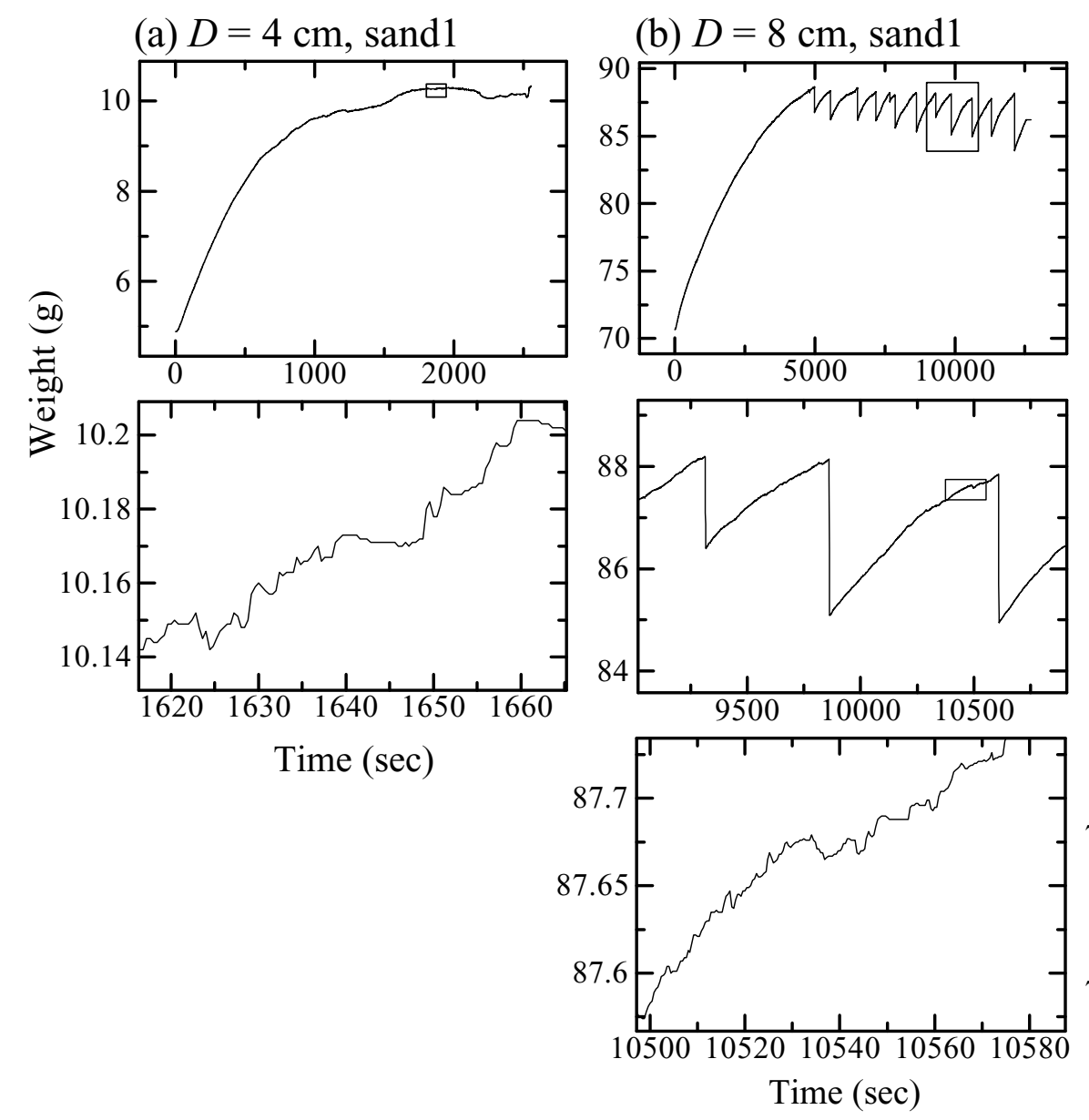

(c) $D=8 \mathrm{~cm}$, sand 2
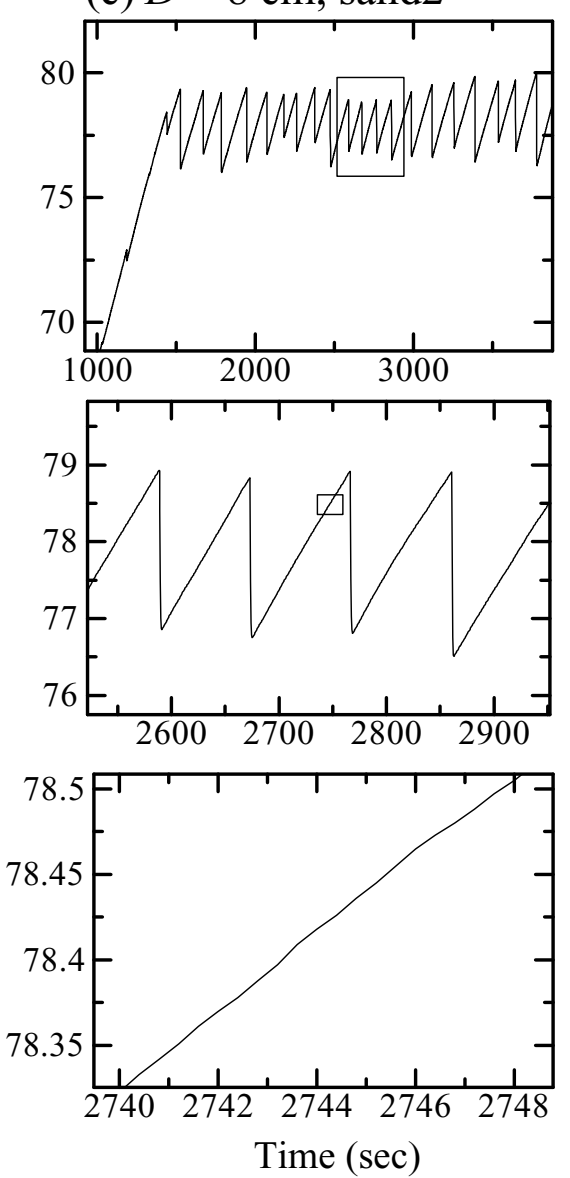

Fig. 4. Fluctuation in mass of sandpiles on (a) a $4 \mathrm{~cm}$ disk, (b) an $8 \mathrm{~cm}$ disk for sand 1 and (c) an $8 \mathrm{~cm}$ disk for sand 2.

nize it as an avalanche, the size of which is defined by $e-e^{\prime}$. From $i$ through $l$, there is a large decrease in weight. This sequence of reduction in weight is regarded as an avalanche.

\section{Results}

The fluctuation in mass of sandpiles is illustrated in Fig. 4. The disk diameter $D$ and the sand used in the experiment are shown at the top of Fig. 4 (a), (b) and (c). The lower figures are the magnified portions of the small boxes in the upper and the middle figures. When disk diameter is small and grain size is relatively large, small avalanches are continuously and randomly occurring as shown in Fig. 4(a). The frequency-size distribution roughly obeys a power law as shown later. However, the increase in disk diameter brings about a drastic transition in the behavior of avalanches. As shown in Fig. 4(b), large avalanches begin to occur on a large $(D=8 \mathrm{~cm})$ disk. They are almost periodic, if not exactly. We notice that this behavior is quite similar to that of characteristic earthquakes. The bottom figure shows that a number of small avalanches are occurring as well in the mass increasing process. Moreover if the ratio of grain size to disk diameter becomes even smaller (Fig. 4(c)), the small avalanches disappear (bottom of Fig. 4(c)) and the large and periodic avalanches become dominant.

Figure 5 shows frequency-size distribution for grain diameters (a) $0.5<d<1.0 \mathrm{~mm}$ (sand 1) and (b) $0.25<d<0.5$ mm (sand 2). The magnitude $M$ of an avalanche is defined by

$$
M=\log W
$$

where $W$ is the size of an avalanche (mass of sand dropping off the disk in an avalanche). Following the standard practice in seismology to use cumulative statistics, the cumulative number of avalanches with a magnitude greater than $M$ is plotted as a function of magnitude. The slight difference in flow rate from experiment to experiment is corrected to the flow rate of $10 \mathrm{mg} / \mathrm{s}$ and the number of avalanches is modified to occurrence rate per minute.

It is clearly seen in Fig. 5(a) that the behavior of avalanches on the small disks obeys a power law (SOC-type behavior), whereas that on the largest disk $(D=8 \mathrm{~cm})$ contains CE-type large avalanches as well as SOC-type small avalanches. It seems that the transition occurs at disk diameter of $8 \mathrm{~cm}$ for sand 1. Furthermore, when sand with smaller diameter is used (Fig. 5(b)), the transition takes place at disk diameter of around $5 \mathrm{~cm}$ and only CE-type large avalanches occurs on the $8 \mathrm{~cm}$ disk.

These facts strongly suggest that the behavior of avalanches is determined solely by the ratio of grain size to disk (or pile) size. Denoting the radius of the disk by $R$, the ratio $\eta$ is given by

$$
\eta=\frac{d}{R}
$$




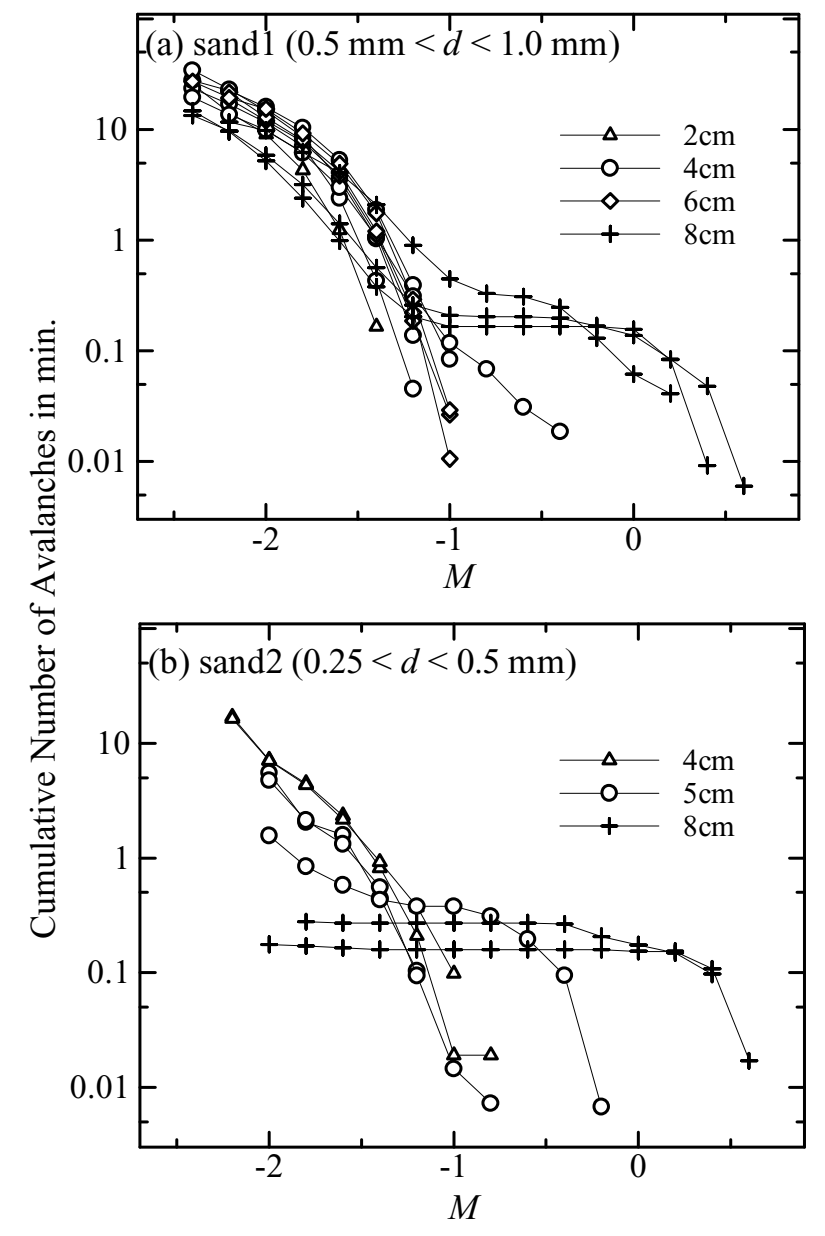

Fig. 5. Frequency-size distribution for grain diameters (a) $0.5<d<$ $1.0 \mathrm{~mm}$ (sand 1) and (b) $0.25<d<0.5 \mathrm{~mm}$ (sand 2).

Since sand 1 begins to exhibit CE-type behavior on the 8cm disk system (Fig. 5(a)), the critical ratio $\eta_{c}$ is roughly estimated as $d / R=0.75 / 40 \simeq 0.02$ assuming the average diameter of sand 1 to be $0.75 \mathrm{~mm}$. Similarly from Fig. 5(b), $\eta_{c}$ for sand 2 is obtained as $d / R=0.4 / 25=0.016 \simeq 0.02$ with the assumption that the average diameter of sand 2 is $0.4 \mathrm{~mm}$.

In summary, the behavior of avalanches changes from SOC-type to CE-type with the increase of disk diameter. The transition occurs when $\eta_{c}$ is around 0.02. This is schematically illustrated in Fig. 6. Note that the frequency-size distribution is very similar to that shown in Fig. 1.

\section{Discussion}

Although our apparatus is poor compared with that used by Held et al. (1990) in the sense that the irregularity of dropping rate and the variation of mass due to input in the duration of avalanches could not be removed, the same property of avalanches was well reconfirmed: when the size of pile is increased, there is a transition from SOC to CE-type behavior. The transition occurs when the ratio of grain size to the disk radius is reduced to about 0.02 . Moreover, we found that if the ratio is further decreased, SOC-type behavior disappears and only CE-type large and periodic avalanches becomes manifest.

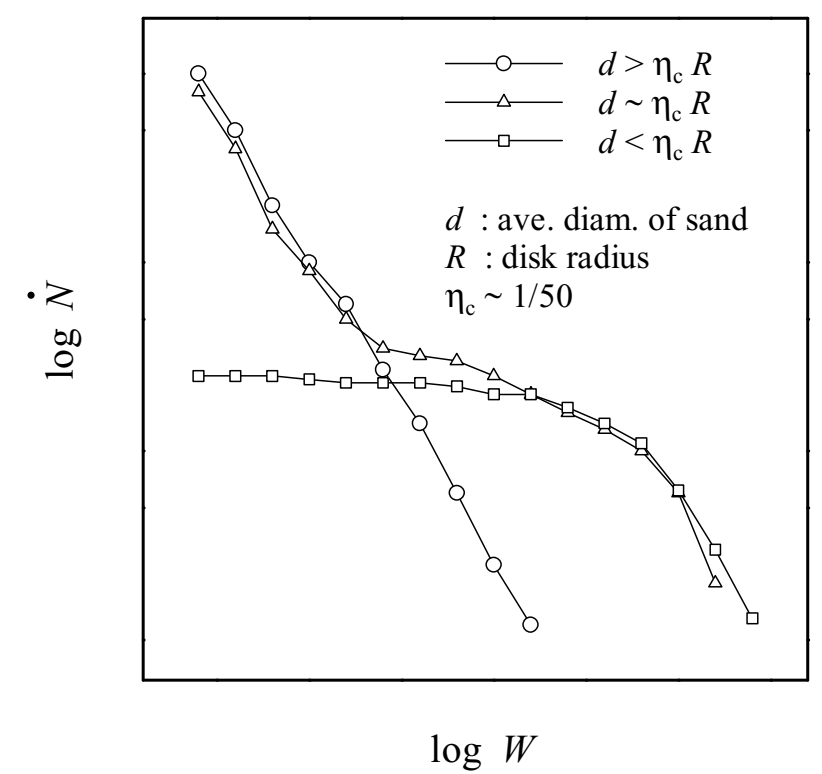

Fig. 6. Schematic illustration of frequency-size distribution of avalanches.

The problem is the underlying mechanism which brings about the transition. One of the ways to approach this problem may be a computer simulation. We performed a preliminary simulation using a one-dimensional cellular automaton model which is similar to the one-dimensional "nonlocal unlimited model" of Kadanoff et al. (1989). The difference is that we add particles onto the left-most cell, whereas Kadanoff et al. added particles to a random site. The model consists of $n$ cells. The height of each cell is defined by the number of particle in the cell. Beginning with all zero height, particles are added one by one onto the left-most cell which is regarded as the apex of a sandpile. Taking into account the fact that the grain does not necessarily rest at the top of the sandpile but rolls down the slope, the particle dropped onto the left-most cell shifts to right by a distance $x$ with a probability

$$
p(x ; a)=a \exp (-a x)
$$

where $a$ is a constant. Cells will collapse and the particles shift to right sites when the height difference between two nearest neighbors becomes a critical level $s_{c}$. The number of particles which may shift to right cells is equal to the height difference. The shift distance for each particle is also given by (3). The particle which shifts beyond the right-most cell will fall out of the system which is regarded as an avalanche. The number of particles which fall off the system by adding one particle is the size of avalanches, the logarithm of which is called "magnitude". The total number of particles in the system is called "weight". A typical example of the results is shown in Fig. 7 for $a=0.3$ and $s_{c}=20$. It is clearly seen that as the number of cell $n$ increases, large avalanches becomes dominant. However, the inlets in Fig. 7(a) show that the enlarged portions of the time chart for $n=10$ cannot be distinguished from that for larger $n$. The system exhibits self-affine fractal nature and there is no drastic transition due to the increase in the number of cell. This is also confirmed by the frequency-magnitude distribution shown in 

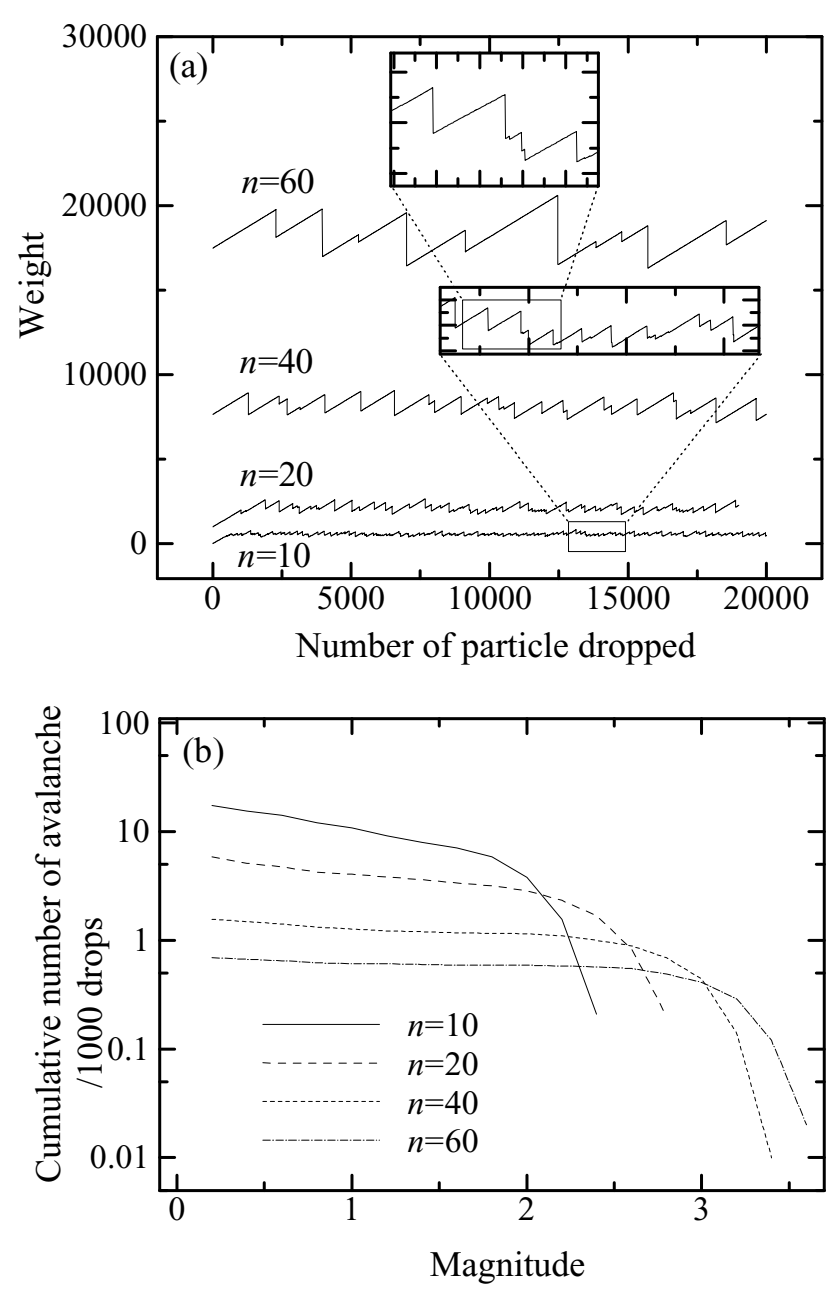

Fig. 7. (a) Time chart of total weight of the cell system and (b) frequency-size distribution obtained by a simulation using a cellular automaton model.

Fig. 7(b). The simple one-dimensional cellular automaton model cannot simulate the transition observed in the experiment. Further the two-dimensional models of Kadanoff et al. (1989) exhibit no drastic transition in a wide range of number of cells. Thus these facts suggest, as a negative example, that for the transition to occur, there should exist a change in some physical conditions.

A group of the University of Chicago proposed a simple explanation for the transition (Liu et al., 1991; Nagel, 1992). They argue that there are two distinct angles of surface slope: the angle of repose $\theta_{r}$ to which the slope angle returns after a large avalanche occurs, and the maximum angle $\theta_{m}$ which is the angle just before a large avalanche occurs (Fig. 8). Between the two angles, the slope is metastable. From their experiments using an open box with sand added from the top and a rotating semicylindrical drum (Jaeger et al., 1989), they found the difference angle

$$
\delta=\theta_{m}-\theta_{r}
$$

to be typically a few degrees. Denoting the length of the slope by $L$, the two angles can be distinguished only when the grain diameter $d$ is small enough to satisfy the condition

$$
d<L \cdot \delta
$$

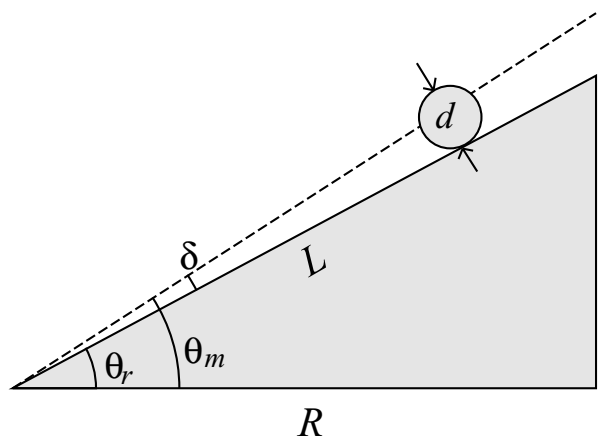

Fig. 8. Two distinct angles: maximum angle $\theta_{m}$ and angle of repose $\theta_{r}$ proposed by Liu et al. (1991) and Nagel(1992) to account for the instability of a particle on the surface of a pile.

because, if (5) is not satisfied, any grain added at any point on the slope will immediately exceed $\theta_{m}$ and the slope is always unstable. Thus the transition occurs when

$$
L(\text { or } R) \simeq \frac{d}{\delta}
$$

For $\delta=2^{\circ}$, we have $L \simeq 30 d$ or $d / L \simeq 0.03$ which roughly agrees with the experimental observation. However this explanation seems to be physically unreasonable because a grain added at any point on the slope does not know how long the slope extends below it. The stability of a grain must be determined by the local physics such as the interaction between a grain and its neighbors.

An alternative explanation may be found in the fact that a conical pile has a stress dip near the center of the pile (Jotaki and Moriyama, 1979; Vanel et al., 1999). Experimental observations of bottom pressure have shown that localized sources (pouring grains from a funnel with a small outlet) yield stress profiles with a clear dip and homogeneous sources (raining from a large sieve) yield no dip. Arching formed by granular material within the pile has been considered to be responsible for the existence of the dip (Jotaki and Moriyama, 1979). This suggests that the spatial stress profiles called stress chains inside the pile is not homogeneous but very much complex, where forces are carried by a small fraction of the total number of grains (Vanel et al., 1999). Thus it is possible that the behavior of avalanches depends on the structure of the stress chains near the surface.

Since the experiments mentioned above were done using grains with diameters much smaller than $\eta_{c}$, we have no experimental evidence that smaller piles have different stress profiles from larger piles. Two-dimensional simulations have been performed by Matuttis (1998) and Schinner et al. (2001). The result shows that large piles have a clear stress dip only when created by a localized source, whereas small piles do not even if a localized source is used. This is consistent with the experimental observations and support the assumption that a transition occurs in stress profile inside and near the surface of the pile as the pile is grown up, although the ratio of grain size to pile size at the transition is not explicitly shown in the papers.

Finally we speculatively argue the maturity of faults. Figure 6 shows that there are three types of avalanche or earthquake behavior: (1) the typical self-organized criticality (G- 
R law), (2) the behavior at the transition and (3) the only CE type behavior. Stirling et al. (1996) made a world-wide survey of strike-slip faults and suggests that the complexities of fault traces evolve with time. According to their idea, a network of small faults with fractal nature gradually evolve into a single smooth fault, by connecting separate faults, breaking barriers and wearing rough fault surfaces. Shimazaki (1999) went one step further and offered an idea of "the almighty earthquake" which is the extreme of the fault maturity. He states "we are somewhere in the evolutionary path, a little bit off the starting point of total chaos but far from the world of the Almighty Earthquake". What we are observing in nature is the mixture of a fractal or chaotic system and a halfmatured system as suggested by the sandpile experiment.

Although we are unfortunately not able to understand the detailed mechanism of the transition at the present stage, it seems very important to further investigate the underlying physics of the transition because it presumably provides us a clue to understand the mechanism of the periodicity of large characteristic earthquakes and may open a way for earthquake prediction.

Acknowledgments. I thank S. Kaketari, M. Nakazawa for their assistance in the experiments and T. Usui for his assistance in the early stage of the numerical simulation. The comments of D. Lavallee and an anonymous reviewer are acknowledged for improving the manuscript. This work was supported by Grant-in-Aid for General Scientific Research (\#09440162) of the Ministry of Education, Science and Culture, Japan and the Earthquake Research Institute of the University of Tokyo Cooperative Research Program (2000-B$01)$.

\section{References}

Ando, M., Source mechanisms and tectonic significance of historical earthquakes along the Nankai trough, Japan, Tectonophys, 27, 119-140, 1975

Bak, P. and C. Tang, Earthquakes as a self-organized critical phenomena, $J$. Geophys. Res., 94, 15635-15637, 1989

Bak, P., C. Tang, and K. Wiesenfeld, Self-organized criticality: An explanation of 1/f noise, Phys. Rev. Lett., 59, 381-384, 1987.

Bak, P., C. Tang, and K. Wiesenfeld, Self-organized criticality, Phys. Rev. A, 38, 364-374, 1988.

Barriere, B. and D. L. Turcotte, Seismicity and self-organized criticality, Phys. Rev. E, 49, 1151-1160, 1994.

Burridge, R. and L. Knopoff, Model and theoretical seismicity, Bull. Seism. Soc. Am., 57, 341-371, 1967

Carlson, J. M. and J. S. Langer, Properties of earthquakes generated by fault dynamic, Phys. Rev. Lett., 62, 2632-2635, 1989a.

Carlson, J. M. and J. S. Langer, Mechanical model of an earthquake fault, Phys. Rev. A, 40, 6470-6484, 1989b.

Evesque, P., Analysis of the statistics of sandpile avalanches using soilmechanics results and concepts, Phys. Rev. A, 43, 2720-2740, 1991.
Geller, R, J., D. D. Jackson, Y. Y. Kagan, and F. Mulargia, Earthquake cannot be predicted, Science, 275, 1616-1617, 1997.

Held, G. A., D. H. Solina, D. T. Keane, W. J. Haag, P. M. Horn, and G. Grinstein, Experimental study of critical-mass fluctuations in an evolving sandpile, Physical Rev. Lett., 65, 1120-1123, 1990.

Ito, K. and M. Matsuzaki, Earthquakes as self-organized critical phenomena, J. Geophys. Res., 95, 6853-6860, 1990.

Jaeger, H. M., C-h., Liu, and S. R. Nagel, Relaxation at the angle of repose, Phys. Rev. Lett., 62, 40-43, 1989.

Jotaki, T. and R. Moriyama, On the bottom pressure distribution of the bulk materials piled with the angle of repose, J. Soc. Powder Technol. Japan, 60, 184-191, 1979 (in Japanese with English abstract and figure caption).

Kadanoff, L. P., S. R. Nagel, L. Wu, and S-m. Zhou, Scaling and universality in avalanches, Phys. Rev. A, 39, 6524-6537, 1989.

Kumagai, H., Time sequence and the recurrence models for large earthquakes along the Nankai trough revisited, Geophys. Res. Lett., 23, 1139 $1142,1996$.

Liu, C-h., H. M. Jaeger, and S. R. Nagel, Finite-size effects in a sandpile, Phys. Rev. A, 43, 7091-7092, 1991.

Matuttis, H.-G., Simulation of the pressure distribution under a twodimensional heap of polygonal particles, Granular Matter, 1, 83-91, 1998.

Nagel, S. R., Instabilities in a sandpile, Rev. Modern Phys., 64, 321-325, 1992.

Nakanishi, H., Cellular-automaton model of earthquakes with deterministic dynamics, Phys. Rev. A, 41, 7086-7089, 1990.

Nakanishi, H., Statistical properties of the cellular-automaton model for earthquakes, Phys. Rev. A, 43, 6613-6621, 1991.

Rosendahl, J., M. Vekic, and J. Kelly, Persistent self-organization of sandpiles, Phys. Rev. E, 47, 1401-1404, 1993.

Rosendahl, J., M. Vekic, and J. E. Rutledge, Predictability of large avalanches on a sandpiles, Phys. Rev. Lett., 73, 537-540, 1994.

Schinner, A., H.-G. Matuttis, T. Akiyama, J. Aoki, S. Takahashi, K. M. Aoki, and K. Kassner, History-dependent structure in granular piles, in Powder and Grains 2001, edited by Swets and Zeitlinger, pp. 499-502, 2001.

Schwartz, D. P. and K. J. Coppersmith, Fault behavior and characteristic earthquakes: Examples from the Wasatch and San Andreas fault zones, $J$ Geophys. Res., 89, 5681-5698, 1984.

Shimazaki, K., The almighty earthquake, Seism. Res. Lett., 70, 147-148, 1999.

Shimazaki, K. and T. Nakata, Time-predictable recurrence model for large earthquakes, Geophys. Res. Lett., 7, 279-282, 1980.

Stirling, M. W., S. G. Wesnousky, and K. Shimazaki, Fault trace complexity, cumulative slip, and shape of the magnitude-frequency distribution for stick-slip faults: a global survey, Geophys. J. Int., 124, 833-868, 1996.

Turcotte, D. L., Self-organized criticality, Rep. Prog. Phys., 62, 1377-1429, 1999.

Vanel, L., D. Howell, D. Clark, R. P. Behringer, and E. Clement, Memories in sand: Experimental tests of construction history on stress distributions under sandpile, Phys. Rev. E, 60, R5040-R5043, 1999.

Wesnousky, S. G., C. H. Scholz, K. Shimazaki, and T. Matsuda, Earthquake frequency distribution and the mechanics of faulting, J. Geophys. Res., 88, 9331-9340, 1983.

N. Yoshioka (e-mail: yoshi@yokohama-cu.ac.jp) 